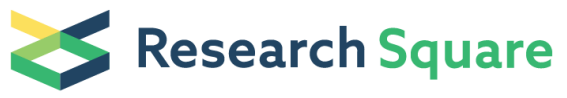 \\ Preprints are preliminary reports that have not undergone peer review. \\ They should not be considered conclusive, used to inform clinical practice, \\ or referenced by the media as validated information.
}

\section{The Relationship Between Paroxysmal Vertigoes and Right-To-Left Shunt: A Large Observational Study}

\section{Kaiming Liu}

Zhejiang University School of Medicine Second Affiliated Hospital

Xiulin Tian

Zhejiang University School of Medicine Second Affiliated Hospital

\section{Wenwu Hong}

Tiantai Hospital of Hangzhou Medical College

Yujin Xiao

Jiaxing Hospital of Traditional Chinese Medicine

\section{Juanyan Chen}

Dongyang People's Hospital

Haiti Jin

Wannan Medical College

\section{Xiaopei Xu}

Zhejiang University School of Medicine Second Affiliated Hospital

\section{Tao Zang}

Tongxiang Second People's Hospital

\section{Liang Zhang}

Zhejiang University School of Medicine Second Affiliated Hospital

\section{Mengxiong Pan}

The First People's Hospital of Huzhou

Xiaodong Zou ( $\sim$ zxd753268@sina.com )

Tongde Hospital Of Zhejiang Province https://orcid.org/0000-0002-4033-3462

\section{Research article}

Keywords: Benign recurrent vertigo, Right-to-left shunt, Paroxysmal vertigoes

Posted Date: November 30th, 2021

DOI: https://doi.org/10.21203/rs.3.rs-1087535/v1

License: @ (1) This work is licensed under a Creative Commons Attribution 4.0 International License. Read Full License 


\section{Abstract \\ Background}

The association between paroxysmal vertigo and right-to-left shunt (RLS) is rarely reported. We investigated the incidence and correlation of RLS in patients with different paroxysmal vertigo diseases.

\section{Methods}

This large observational study included patients with paroxysmal vertigo from seven different hospitals in China from 2017 to 2021 (NCT04939922). Migraine patients within the same period were included for comparison. Demographic data and medical history were collected, contrast transthoracic echocardiography (CTTE) was performed, and the clinical features, dizziness handicap inventory, and incidence of RLS in each group were recorded.

\section{Results}

This study used a consecutive sampling of 4536 patients from seven centres, and a total of 2751 patients were enrolled. The proportion of RLS in patients with migraine with aura (MA), migraine without aura (MoA), vestibular migraine (VM) with headache, VM without headache, and benign recurrent vertigo (BRV) was significantly higher than that in patients with benign paroxysmal positional vertigo (BPPV), Meniere's disease (MD), and vestibular paroxysmia (VP) $(P<0.05)$. There was no statistical difference between the frequency of RLS in patients with $\mathrm{BRV}$ and those with $\mathrm{MoA}(P=0.931), \mathrm{MA}(\mathrm{P}=0.997), \mathrm{VM}$ with migrainous headache $(P=0.787)$, and VM without migrainous headache $(P=0.754)$. There was a positive correlation between the RLS grade and the dizziness handicap inventory scores of VM and BRV patients $(P<0.01)$.

\section{Conclusions}

RLS was significantly associated with BRV and VM. RLS may be involved in the pathogeneses of BRV and VM and may serve as a reference index for the differential diagnosis of central and peripheral vertigo.

\section{Trial registration:}

CHRS, NCT04939922, registered 14 June 2021- retrospectively registered, https://register.clinicaltrials.gov

\section{Background}

Paroxysmal vertigo resulting from different diseases has distinct clinical symptoms and signs. However, significant overlaps exist in the clinical symptoms of different vestibular diseases, such as vestibular migraine (VM), which may have manifestations similar to Meniere's Disease (MD) or benign paroxysmal positional vertigo (BPPV) [1]. Furthermore, some VM patients may have no headache, while MD may be accompanied by migraine, photophobia, phonophobia, and other symptoms. This increases the difficulty in distinguishing these diseases from each other [2,3]. Benign recurrent vertigo (BRV) was first described by Slater and is defined as a group of clinical syndromes with recurrent vertigo without nervous system and cochlear symptoms [4]. At present, there are no recognised diagnostic criteria for paroxysmal vertigo; thus, diagnosis by exclusion is often used [5]. Follow-up studies have shown that BRV is closely associated with migraine as 51$87 \%$ of BRV cases are comorbid with migraine [6,7]. This proportion of BRV meets the description of definite VM or possible VM. BRV and VM differ in terms of the definition and diagnostic criteria. BRV can have no headache and migraine, while VM requires at least a history of migraine or migraine-like symptoms [8]. Given that BRV and VM have no specific physical or auxiliary examination findings, clinical diagnosis is often a challenge. Therefore, identifying anatomic features related to certain forms of paroxysmal vertigo that may aid in establishing diagnosis may be helpful. This may also help in determining the pathogenesis of diseases with similar clinical symptoms.

Many studies have found that the incidence of patent foramen ovale (PFO) in migraine patients is significantly higher than that in normal people $[9,10]$. The relationship between PFO and migraine has been studied and is a major concern [11]. Vestibular migraine is considered to be related to vestibular symptoms caused by migraine mechanisms, and its pathogenesis may be related to ion channel defects, enhanced cortical excitability, and central sensitisation, which are caused by genetic susceptibility [12, 13]. PFO is the most common cause of right-toleft shunt (RLS). RLS refers to the potentially abnormal channels between the left and right atrium and ventricle, or systemic circulation and 
pulmonary circulation. Based on its location, RLS can be categorised as intracardiac shunt and extracardiac shunt. The former includes PFO, while the latter includes pulmonary arteriovenous malformation and patent ductus arteriosus, among others. Current research rarely focuses on the relationship between RLS and paroxysmal vertigo. Diagnostic tests used for detecting RLS mainly include contrast transcranial Doppler, contrast transthoracic echocardiography (CTTE), and contrast transoesophageal echocardiography. Given that cTTE is easy to operate and has high sensitivity and specificity [14], we chose this method to investigate the incidence of paroxysmal vertigo (VM with headache, VM without headache, BRV, MD, BPPV, and VP) in adults with RLS compared to migraine patients, thus evaluating the correlation between RLS and different types of paroxysmal vertigo diseases.

\section{Methods}

\section{Study design and patient population}

The patients included in the study were mainly from the headache and vertigo clinics of seven different hospitals in China from July 2017 to July 2021 (ClinicalTrial.gov ID: NCT04939922). The study used a consecutive sampling of patients $(n=4536)$ with migraine or paroxysmal vertigo aged over 18 years. Patients were screened for VM, pure BRV, definite MD (dMD), BPPV, vestibular paroxysmia (VP), migraine with aura (MA), and migraine without aura (MoA) and, if positive, were included in the study. Patients who were diagnosed with probable migraine, psychiatric vestibular disorder, probable MD, probable VP, functional vestibular disorder, vertebral basilar transient ischemic attack (TIA), migraine with brain stem aura, episodic ataxia, cerebellopontine angle tumour, vestibular epilepsy, superior canal dehiscence/perilymphatic fistula, and other paroxysmal vertigo patients who did not meet the diagnostic criteria of all other vertigo diseases were excluded. A flowchart of patient inclusion and exclusion is depicted in Figure 1.

The inclusion criteria for VM patients met the definite and probable diagnostic criteria for VM established by the International Barany Society and the International Headache Society [8].

The inclusion criteria for BRV in this study were based on the exclusive diagnostic method proposed in the literature [15], and a personal history of no migraine headache, visual aura, photophobia, and phonophobia (to distinguish and exclude definite VM and probable VM), which we defined as pure BRV here (Table 1).

Table 1

Inclusion criteria for pure benign recurrent vertigo

\section{More than 2 attacks of spontaneous rotational vertigo that does not occur during the head movements or positional changes}

No associated migrainous headaches or auditory symptoms during or between the attacks

No associated photophobia and phonophobia

No associated visual aura

No associated focal neurologic symptom during the attack or afterward suggesting episodic ataxia, transient ischemic attack, or vestibular epilepsy

No evidence of peripheral vestibulopathy on head-impulse, caloric, and rotatory tests

No asymmetric hearing impairment documented in pure-tone audiometry

No lesions on brain MRIs responsible for the benign recurrent vertigo

No history of disorders that may explain the recurrent vertigo

Not better accounted for by another vestibular disorder, including compensated vestibular neuritis, vestibular migraine, Meniere's disease, benign paroxysmal positional vertigo, and vestibular paroxysmia

The selection criteria for dMD, BPPV, and VP patients were in accordance with the diagnostic criteria established by the Barany Association, and the selection criteria for migraines were consistent with the diagnostic criteria of The International Classification of Headache Disorders, $3^{\text {rd }}$ edition [16-19]. As RLS is strongly related to migraines with auras, migraines were further divided into MA and MoA, and VM was further divided into VM with and without headache. All VP patients selected in this study were responsive to carbamazepine treatment. All patients were diagnosed by two experienced neurologists to reduce bias.

The patients included underwent cTTE and were assigned into eight groups based on diagnosis: VM with migrainous headache, VM without migrainous headache, pure BRV, dMD, BPPV, VP, MA, and MoA. This study was approved by the ethics committees of the participating centres, and all patients signed informed consent forms. 


\section{Contrast transthoracic echocardiography inspection method}

While the patients were in the supine position, venous access was established by conventional elbow venipuncture, and a three-way tube was connected to the puncture site. The contrast medium was formulated after $8 \mathrm{~mL}$ saline, $1 \mathrm{~mL}$ venous blood, and $1 \mathrm{~mL}$ air was fully injected and concussed through the tee tube. The contrast was quickly injected while the patient was at rest and while doing Valsalva manoeuvre. The apical four-chamber section was selected, and the number of microbubble signals in the left cardiac system was observed within 10 cardiac cycles after the right cardiac system was filled with microbubble signals. RLS of the heart was considered when there was more than one microbubble in the left cardiac cavity.

The RLS was graded quantitatively according to the number of microbubbles in the left ventricular cavity after the Valsalva manoeuvre. The grading standard are as follows: grade 0 - no microbubbles in the left cardiac cavity and no RLS; grade I - 1 to 10 microbubbles/frame were seen in the left ventricular cavity and a small amount of RLS; grade II - 10 to 30 microbubbles/frame were seen in the left cardiac cavity and a medium amount of RLS; grade III - more than 30 microbubbles/frame were seen in the left ventricular cavity, or the left ventricular cavity was almost full of microbubbles [20].

RLS from PFO was considered when microbubbles were found within 3-5 cardiac cycles, while RLS from pulmonary arteriovenous malformations was considered when microbubbles were found in more than five cardiac cycles during the cTTE examination [21].

\section{Dizziness Handicap Inventory}

The quality of life measure for vestibular disorders was assessed using the 25-item Dizziness Handicap Inventory (DHI), which has three response categories: the functional, emotional, and physical aspects of life [22]. The total score ranges from 0 (not handicapped) to 100 (severely handicapped).

\section{Statistical analysis}

Data measurements assumed a normal distribution and are expressed as $\mathrm{x} \pm \mathrm{s}$. We used the $\chi^{2}$ test and t-test (or Mann-Whitney $\mathrm{U}$-test) to compare categorical and continuous variables. Differences between more than two groups were analysed using one-way ANOVA.

Spearman's correlation coefficient was used to analyse the correlation between the DHI and RLS grades. The level of statistical significance was set at $\mathrm{P}<0.05$. All analyses were performed using the SPSS version 20.

\section{Results}

\section{Patient demographic and clinical characteristics at baseline}

Within the time frame, a total of 2751 patients completed cTTE at the outpatient departments of seven participating centres. The mean age was $43.16 \pm 14.58$ years, and $65.18 \%$ were female. The demographics and clinical characteristics of the patients are summarised in Table 2. 
Table 2

Demographic and clinical characteristics of patients at baseline

\begin{tabular}{|c|c|c|c|c|c|c|c|c|}
\hline & $\begin{array}{l}\text { MoA } \\
\text { without } \\
\text { vertigo } \\
(n=1399)\end{array}$ & $\begin{array}{l}\text { MA } \\
\text { without } \\
\text { vertigo } \\
(n=216)\end{array}$ & $\begin{array}{l}\text { VM with } \\
\text { migrainous } \\
\text { headache } \\
(n=410)\end{array}$ & $\begin{array}{l}\text { VM without } \\
\text { migrainous } \\
\text { headache } \\
(n=119)\end{array}$ & $\begin{array}{l}\text { MD } \\
(n=148)\end{array}$ & $\begin{array}{l}\text { BPPV } \\
(n=331)\end{array}$ & $\begin{array}{l}\text { VP } \\
(n=57)\end{array}$ & $\begin{array}{l}\text { BRV } \\
(n=71)\end{array}$ \\
\hline $\begin{array}{l}\text { Sex (female, } \\
\%)\end{array}$ & 73.27 & 75.46 & 76.83 & 72.27 & 57.43 & 64.65 & 59.65 & 71.83 \\
\hline $\begin{array}{l}\text { Age } \\
(\text { mean } \pm S D)\end{array}$ & $39.85 \pm 15.47$ & $37.7 \pm 15.41$ & $42.67 \pm 12.56$ & $36.67 \pm 14.99$ & $52.35 \pm 7.28$ & $56.13 \pm 10.48$ & $53.74 \pm 6.15$ & $50.7 \pm 18.93$ \\
\hline \multicolumn{9}{|l|}{$\begin{array}{l}\text { Duration of } \\
\text { attacks }\end{array}$} \\
\hline$<1 \mathrm{~min}$ & & & 45 & 17 & 0 & 331 & 47 & 7 \\
\hline$<5 \min$ & NA & NA & 94 & 15 & 0 & 0 & 10 & 13 \\
\hline$<1 \mathrm{~h}$ & & & 128 & 29 & 85 & 0 & 0 & 26 \\
\hline$<24 h$ & & & 118 & 41 & 63 & 0 & 0 & 18 \\
\hline$>24 \mathrm{~h}$ & & & 25 & 17 & 0 & 0 & 0 & 7 \\
\hline $\begin{array}{l}\text { Migrainous } \\
\text { headache }\end{array}$ & 1399 & 216 & 410 & 0 & 0 & 0 & 0 & 0 \\
\hline Aura & 0 & 216 & 53 & 16 & 0 & 0 & 0 & 0 \\
\hline Photophobia & 992 & 156 & 386 & 107 & 0 & 2 & 1 & 3 \\
\hline Phonophobia & 984 & 167 & 389 & 110 & 1 & 0 & 0 & 1 \\
\hline $\begin{array}{l}\text { Photophobia } \\
\text { \& } \\
\text { phonophobia }\end{array}$ & 961 & 144 & 372 & 101 & 0 & 0 & 0 & 0 \\
\hline
\end{tabular}

\section{Right-to-left shunt in patients from different groups}

A total of $61.98 \%$ of RLS-positive patients were detected by cTTE after the Valsalva manoeuvre. The proportion of RLS in patients with BRV and VM with and without migrainous headache was significantly higher than that in patients with MD, BPPV, and VP (P<0.05). There was no statistical difference between the frequency of RLS in patients with BRV and those with MoA ( $P=0.931), M A(P=0.997), V M$ with migrainous headache $(P=0.787)$, and VM without migrainous headache $(P=0.754)$ (Table 3 and Figure 2). 
Table 3

Right-to-left shunt and Dizziness Handicap Inventory in patients from different groups

\begin{tabular}{|c|c|c|c|c|c|c|c|c|}
\hline & $\begin{array}{l}\text { MoA without } \\
\text { vertigo } \\
(n=1399)\end{array}$ & $\begin{array}{l}\text { MA } \\
\text { without } \\
\text { vertigo } \\
(n=216)\end{array}$ & $\begin{array}{l}\text { VM with } \\
\text { migrainous } \\
\text { headache } \\
(n=410)\end{array}$ & $\begin{array}{l}\text { VM without } \\
\text { migrainous } \\
\text { headache } \\
(n=119)\end{array}$ & $\begin{array}{l}\text { MD } \\
(n=148)\end{array}$ & $\begin{array}{l}\text { BPPV } \\
(n=331)\end{array}$ & $\begin{array}{l}\text { VP } \\
(n=57)\end{array}$ & $\begin{array}{l}\text { BRV } \\
(n=71)\end{array}$ \\
\hline RLS (\%) & 65.33 & 81.01 & 77.07 & 76.47 & 27.03 & 25.38 & 26.31 & 73.24 \\
\hline PFO & 828 & 153 & 292 & 79 & 35 & 73 & 14 & 46 \\
\hline PAVM & 57 & 12 & 19 & 9 & 2 & 8 & 1 & 4 \\
\hline $\begin{array}{l}\text { PFO \& } \\
\text { PAVM }\end{array}$ & 29 & 10 & 5 & 3 & 3 & 3 & 0 & 2 \\
\hline \multicolumn{9}{|l|}{$\begin{array}{l}\text { Grading } \\
\text { of RLS }\end{array}$} \\
\hline 0 & 485 & 41 & 94 & 28 & 108 & 247 & 42 & 19 \\
\hline I & 263 & 33 & 68 & 17 & 17 & 40 & 7 & 12 \\
\hline II & 257 & 54 & 106 & 34 & 14 & 30 & 3 & 18 \\
\hline III & 394 & 88 & 142 & 40 & 9 & 14 & 5 & 22 \\
\hline $\mathrm{DHI}$ & NA & NA & $46.33 \pm 10.61$ & $47.46 \pm 9.40$ & $51.26 \pm 9.48$ & $47.37 \pm 13.42$ & $50.69 \pm 6.87$ & $46.87 \pm 10.37$ \\
\hline
\end{tabular}

\section{Right-to-left shunt in benign recurrent vertigo patients}

The proportion of patients with RLS in seven patients with BRV lasting less than one minute was similar to those lasting for more than one minute $(71.43 \%$ vs $73.44 \%)$. This was significantly higher than in VP (26.31\%) and BPPV (25.38\%). The proportion of RLS in BRV patients aged $<50$ years and those ages $\geq 50$ years was similar (73.08\% vs $73.68 \%$ ), and the difference was not statistically significant (Figure 3 ).

\section{Association between right-to-left shunt grade and dizziness handicap index scores}

Based on the semi-quantitative classification of RLS, the DHI scores of grades $0, \mathrm{I}, \mathrm{II}$, and III of patients from different groups are shown in Table 3. The RLS grade and DHI scores in patients with VM with $(r=0.361,95 \% \mathrm{Cl} 0.27-0.44)$, without headache ( $r=0.528,95 \% \mathrm{Cl} 0.38-0.65)$ and BRV $(r=0.565,95 \% \mathrm{Cl} 0.38-0.71)$ are shown in Figures 4A-C, respectively. The DHI score and RLS grade of all patients with VM or BRV $(r=0.412,95 \% \mathrm{Cl} 0.34-0.48)$ are presented in Figure 4D. In VM and BRV patients, DHI scores increased with increasing RLS grade, and there was a positive correlation between them.

\section{Discussion}

VM is a separate disease entity with a genetic predisposition to recurrent dizziness or vertigo, with or without headache [23]. When presented with positional vertigo, VM and BPPV are difficult to distinguish. The difference lies in the atypical nystagmus of VM, which is usually not aligned with a single semicircular canal, while the nystagmus of BPPV induced by positional testing is synchronised with the symptoms and has a characteristic short duration, brief latency, and fatigability $[2,24]$. VM and BRV may also show spontaneous vertigo, similar to MD. The auditory symptoms of VM and BRV are mild, whereas the hearing loss of MD patients is gradually aggravated with disease progression [16]. When the duration of spontaneous vertigo in VM or BRV is less than one minute, it can be similar to VP. VP is caused by vascular compression of the vestibular nerve, with attacks ranging from 30 times a day to several times a year, without headache. Carbamazepine is an effective treatment for VP [18]. However, the selected VM patients in this study were effectively treated with flunarizine, which increased the reliability of VM diagnosis. Since the subjects were all older than 18 years, the possibility of BPV in children was excluded.

Common paroxysmal vertigo can be categorised based on aetiology: central and peripheral aetiologies. The most common central causes are VM and transient ischemic attack, and the common peripheral causes are BPPV, MD, and VP. However, the classification of these four diseases does not cover all paroxysmal vertigo. Those with unknown causes of recurrent vertigo are classified as BRV, with a high incidence 
in the clinic. The BRV selected in previous clinical studies did not rule out a history of migraine and migraine-related symptoms, so a considerable number of BRV may have met the diagnostic criteria for VM $[6,7]$. To avoid confusion, the selected BRV in this study only showed paroxysmal vertigo, that is, pure BRV. The selected VM patients without headache could be differentiated from pure BRV by its migraine characteristics, such as photophobia, phonophobia, or visual aura. A recent study reported for the first time a group of patients with paroxysmal vertigo with interictal headshaking nystagmus (HSN) [15]. The authors believe central lesions lead to a prolonged time constant, strong HSN, and perverted nystagmus through activated and asymmetric central velocity storage mechanisms. Our results demonstrated that BRV had a significantly higher proportion of RLS than peripheral vertigo diseases such as MD, BPPV, and VP. Although there were no central symptoms of photophobia, phonophobia, or visual aura, we speculate that pure BRV may be central vertigo without these central symptoms and is worthy of further study and discussion.

There is a dose-effect relationship between PFO and migraine. Larger PFO, permanent PFO, and anatomical variation of PFO can aggravate RLS, which is associated with migraine attacks [11]. Meanwhile, PFO is more associated with MA than MoA. The correlation between RLS and migraines does not imply causality, and the mechanism between the two is not clear. The possible mechanism is that vasoactive substances and microemboli from the venous system directly enter systemic circulation without passing through the pulmonary circulation, causing transient hypoperfusion in the area supplied by the cerebral arteries or cortical spreading depression, activating the trigeminal neurovascular system, and causing migraine attacks $[25,26]$. The initiating mechanism of VM is considered to be similar to migraine because RLS not only induces migraine but also causes vertigo by inducing plasma extravasation in the inner ear [27]. Microemboli or vasoactive substances may induce CSD and activate the caudal parabrachial nucleus, which receives both trigeminal receptors and vestibular nerve afferents, resulting in simultaneous vestibular symptoms and migraine symptoms $[1,28]$. Therefore, we speculate that RLS may be involved in the pathogenesis of VM.

Many studies have found that BRV is highly correlated with migraine or VM and may have similar pathogenesis $[6,29,30]$. However, some studies have suggested otherwise [31]. Whether BRV is a separate entity is still controversial, but a considerable number of BRVs are free from inner ear dysfunction and migraine and therefore do not develop into MD and VM, suggesting that there may be different mechanisms in this aspect of BRV [7]. This study showed that the proportion of RLS in patients with BRV, VM, and migraine was significantly higher than those with peripheral vertigo disease, suggesting that RLS might play an important role in the pathogenesis of BRV, VM, and migraine. The current literature remains discordant as to whether a linkage exists between RLS grade and the degree of vertigo. We found that the DHI score increased with an increase in the RLS shunt, suggesting that RLS plays an important role in the degree of vertigo and quality of life of vestibular disorders. The proportions of RLS in patients with BRV and VM were similar to those of migraine, suggesting that the abovementioned mechanisms may share some features in the pathogenesis of migraine. When the related mechanism of migraine only involves the vestibular system, it can manifest as pure BRV, whereas when the vestibular system and trigeminal neurovascular system are simultaneously involved, it can manifest as VM. When only the trigeminal neurovascular system is involved, the symptoms may include migraine, suggesting that BRV and VM may be subtypes or equivalents of migraine. VM and pure BRV can be unified in the migraine category. In this study, the RLS of patients with BPPV and VP was significantly lower than that of patients with migraine, suggesting that RLS was not involved in the pathogenesis of these peripheral vertigo diseases. RLS can play a role in the differential diagnosis of paroxysmal vertigo. In the future, for refractory VM and BRV patients, the evaluation of RLS and the intervention and treatment of RLS are worthy of further exploration.

Previous literature has predominantly defined the duration of BRV as more than $1 \mathrm{~min}$ or even more than $5 \mathrm{~min}$, mainly distinguishing it from BPPV and VP, which are less than $1 \mathrm{~min}$. There are few reports of a BRV of less than $1 \mathrm{~min}$. A previous study found six cases of BRV with migraine that were less than $1 \mathrm{~min}$, and only one case of BRV without migraine was less than 1 minute [6]. In clinical practice, there are truly spontaneous recurrent vertigoes lasting less than $1 \mathrm{~min}$. These patients do not meet the diagnostic criteria for BPPV and VP, and they do not meet the diagnostic criteria for VM. The duration of BRV was not specifically limited in this study. The proportion of RLS between the patients with BRV lasting less and more than 1 minute was similar, and significantly higher than that of VP and BPPV. Thus, strictly defining the duration for diagnosis of BRV may be debatable, and the evaluation of RLS might help to provide a reference for BRV diagnosis.

Due to the low probability of migraine onset after 50 years of age, patients with headache-free BRV after the age of 50 have a very low probability of developing VM, while patients with BRV before the age of 50 still have the possibility of progressing to VM. Therefore, we regarded age 50 as the threshold for the intragroup (subgroup) analysis of BRV. The results showed that the proportion of RLS in patients with BRV before and after 50 years of age was not significantly different and was similar to that of migraine. This may be related to the fact that BRV rarely evolves into VM [32].

With the insight into VM and the gradual clarification of its concept, there is an overlap between the classical concepts of BRV and VM, leading to ambiguity in the concept of BRV; thus, its effective use in clinics is difficult. In this study, pure BRV was proposed for the first time, and it can be effectively distinguished from VM in the absence of migraine-related characteristics. Pure BRV can also be effectively

Page $7 / 14$ 
distinguished from peripheral vertigo, such as MD. Therefore, the concept of pure BRV is helpful for clinicians to further study the mechanism, clinical characteristics, and prognosis of the disease. The concept and classification of VM and BRV in the future are worthy of further discussion.

There are several limitations to our study. First, because TIA, persistent postural-perceptual dizziness, epileptic vertigo, and paroxysmal ataxia were excluded from the selection criteria of BRV in this study, these paroxysmal vertigoes were not compared with RLS. Second, BRV with HSN has been reported to originate from the central nervous system, and in this study, BRV was not subdivided according to whether it was associated with HSN.

\section{Conclusions}

In conclusion, this is a large multicentre study and the findings suggest that RLS differs in different types of paroxysmal vertigo. The incidence of RLS in pure BRV and VM was similar but significantly higher than that in MD, BPPV, and VP. There was a positive correlation between RLS grading and DHI among patients with BRV and VM. The existence of RLS is valuable in the etiological diagnosis of paroxysmal vertigo and may be used as a reference for the differential diagnosis of paroxysmal vertigo.

\section{List Of Abbreviations}

$\begin{array}{ll}\text { BPPV } & \text { Benign paroxysmal positional vertigo } \\ \text { BRV } & \text { Benign recurrent vertigo } \\ \text { CTTE } & \text { Contrast transthoracic echocardiography } \\ \text { DHI } & \text { Dizziness Handicap Inventory } \\ \text { dMD } & \text { Definite Meniere's disease } \\ \text { HSN } & \text { Headshaking nystagmus } \\ \text { MA } & \text { Migraine with aura } \\ \text { MD } & \text { Meniere's disease } \\ \text { MoA } & \text { Migraine without aura } \\ \text { PAVM } & \text { Pulmonary arteriovenous malformation } \\ \text { PFO } & \text { Patent foramen ovale } \\ \text { RLS } & \text { Right-to-left shunt } \\ \text { TIA } & \text { Transient ischemic attack } \\ \text { VM } & \text { Vestibular migraine } \\ \text { VP } & \text { Vestibular paroxysmia } \\ \text { M } & \end{array}$

\section{Declarations}

Ethics approval and consent to participate

The study procedure was approved by the ethics committee of the Second Affiliated Hospital, School of Medicine, Zhejiang University. All patients signed informed consent forms.

\section{Consent for publication}

Not applicable

\section{Availability of data and materials}


All the data supporting our findings is contained within the manuscript

\section{Competing interests}

None

\section{Funding}

We acknowledge funding support from the Zhejiang Provincial Natural Science Foundation of China (Grant No. LY19H090025, Grant No. LQ15H090003), National Natural Science Foundation of China (Grant No. 82171561, Grant No. 82174132). The funders had no role in the design of this study or in the interpretation or presentation of its results.

\section{Authors' contributions}

$\mathrm{KL}$ conducted the design and conceptualization of the study, interpretation of the data, and revising the manuscript. $\mathrm{XZ}$ wrote and designed the manuscript. XT analyzed and interpreted the data. WH, YX, JC, HJ, XX, TZ, LZ, and MP collected and organized the data.

\section{Acknowledgements}

None

\section{References}

1. Furman JM, Marcus DA, Balaban CD (2013) Vestibular migraine: clinical aspects and pathophysiology. Lancet Neurol 12:706-715. DOI: 10.1016/S1474-4422(13)70107-8

2. Stolte B, Holle D, Naegel S, Diener HC, Obermann M (2015) Vestibular migraine. Cephalalgia 35:262-270. DOI: $10.1177 / 0333102414535113$

3. Radtke A, Lempert T, Gresty MA, Brookes GB, Bronstein AM, Neuhauser H (2002) Migraine and Meniere's disease: is there a link? Neurology 59:1700-1704. DOI: 10.1212/01.wnl.0000036903.22461.39

4. Slater R (1979) Benign recurrent vertigo. J Neurol Neurosurg Psychiatry 42:363-367. DOI: 10.1136/jnnp.42.4.363

5. van Esch BF, van Wensen E, van der Zaag-Loonen HJ, Benthem PPGV, van Leeuwen RB (2017) Clinical characteristics of benign recurrent vestibulopathy: clearly distinctive from vestibular migraine and Meniere's disease? Otol Neurotol 38:e357-e363. DOI: 10.1097/MA0.0000000000001553

6. Cha YH, Lee H, Santell LS, Baloh RW (2009) Association of benign recurrent vertigo and migraine in 208 patients. Cephalalgia 29:550555. DOI: 10.1111/j.1468-2982.2008.01770.x

7. Brantberg K, Baloh RW (2011) Similarity of vertigo attacks due to Meniere's disease and benign recurrent vertigo, both with and without migraine. Acta Oto-laryngol 131:722-727. DOI: 10.3109/00016489.2011.556661

8. Lempert T, Olesen J, Furman J, Waterston J, Seemungal B, Carey J, Bisdorff A, Versino M, Evers S, Newman-Toker D (2012) Vestibular migraine: diagnostic criteria. J Vestib Res 22:167-172. DOI: 10.3233/VES-2012-0453

9. Lip PZ, Lip GY (2014) Patent foramen ovale and migraine attacks: a systematic review. Am J Med 127:411-420. DOI: 10.1016/j.amjmed.2013.12.006

10. Tariq N, Tepper SJ, Kriegler JS (2016) Patent foramen ovale and migraine: closing the debate - a review. Headache 56:462-478. DOI: 10.1111/head.12779

11. Liu K, Wang BZ, Hao Y, Song S, Pan M (2020) The correlation between migraine and patent foramen ovale. Front Neurol 11:543485. DOI: 10.3389/fneur.2020.543485

12. Baloh RW (2020) Vestibular migraine I: Mechanisms, diagnosis, and clinical features. Semin Neurol 40:76-82. DOI: 10.1055/s-00393402735

13. Dieterich M, Obermann M, Celebisoy N (2016) Vestibular migraine: the most frequent entity of episodic vertigo. J Neurol 263 Suppl 1:S82-S89. DOI: 10.1007/s00415-015-7905-2

14. Mojadidi MK, Winoker JS, Roberts SC, Msaouel P, Gevorgyan R, Zolty R (2014) Two-dimensional echocardiography using second harmonic imaging for the diagnosis of intracardiac right-to-left shunt: a meta-analysis of prospective studies. Int $\mathrm{J}$ Cardiovasc Imaging 30:911-923. DOI: 10.1007/s10554-014-0426-8

15. Lee SU, Choi JY, Kim HJ, Kim JS. (2018) Recurrent spontaneous vertigo with interictal headshaking nystagmus. Neurology 90:e2135e2145-e45. DOI: 10.1212/WNL.0000000000005689 
16. Lopez-Escamez JA, Carey J, Chung WH, Goebel JA, Magnusson M, Mandalà M, Newman-Toker DE, Strupp M, Suzuki M, Trabalzini F, Bisdorff A, Classification Committee of the Barany Society, Japan Society for Equilibrium Research, European Academy of Otology and Neurotology (EAONO), Equilibrium Committee of the American Academy of Otolaryngology-Head and Neck Surgery (AAO-HNS), Korean Balance Society (2015) Diagnostic criteria for Meniere's disease. J Vestib Res 25:1-7. DOI: 10.3233/VES-150549

17. von Brevern M, Bertholon P, Brandt T, Fife T, Imai T, Nuti D, Newman-Toker D (2015) Benign paroxysmal positional vertigo: diagnostic criteria. J Vestib Res 25:105-117. DOI: 10.3233/VES-150553

18. Strupp M, Lopez-Escamez JA, Kim JS, Straumann D, Jen JC, Carey J, Bisdorff A, Brandt T (2016) Vestibular paroxysmia: diagnostic criteria. J Vestib Res 26:409-415. DOI: 10.3233/VES-160589

19. Headache Classification Committee of the International Headache S. The International Classification of Headache Disorders (2013), 3rd ed. (beta version). Cephalalgia 33:629-808

20. Zhao E, Wei Y, Zhang Y, Zhai N, Zhao P, Liu B (2015) A comparison of Transthroracic Echocardiograpy and transcranial Doppler with contrast agent for detection of patent foramen ovale with or without the valsalva maneuver. Med (Baltim) 94:e1937. DOI: 10.1097/MD.0000000000001937

21. Freeman JA, Woods TD (2008) Use of saline contrast echo timing to distinguish intracardiac and extracardiac shunts: failure of the 3- to 5-beat rule. Echocardiography 25:1127-1130. DOI: 10.1111/j.1540-8175.2008.00741.x

22. Jacobson GP, Newman CW (1990) The development of the Dizziness Handicap Inventory. Arch Otolaryngol Head Neck Surg 116:424427. DOI: 10.1001/archotol.1990.01870040046011

23. Huang TC, Wang SJ, Kheradmand A (2020) Vestibular migraine: an update on current understanding and future directions. Cephalalgia 40:107-121. DOI: 10.1177/0333102419869317

24. von Brevern M, Radtke A, Clarke AH, Lempert T (2004) Migrainous vertigo presenting as episodic positional vertigo. Neurology 62:469472. DOI: 10.1212/01.wnl.0000106949.55346.cd

25. Beda RD, Gill EA, Jr (2005) Patent foramen ovale: does it play a role in the pathophysiology of migraine headache? Cardiol Clin 23:9196. DOI: $10.1016 /$ j.ccl.2004.10.004

26. Morelli N, Rota E (2014) Migraine and patent foramen ovale: barking up the wrong tree? Front Neurol 5:99. DOI: 10.3389/fneur.2014.00099

27. Koo JW, Balaban CD (2006) Serotonin-induced plasma extravasation in the murine inner ear: possible mechanism of migraineassociated inner ear dysfunction. Cephalalgia 26:1310-1319. DOI: 10.1111/j.1468-2982.2006.01208.x

28. Marano E, Marcelli V, Di Stasio E, Bonuso S, Vacca G, Manganelli F, Marciano E, Perretti A (2005) Trigeminal stimulation elicits a peripheral vestibular imbalance in migraine patients. Headache 45:325-331. DOI: 10.1111/j.1526-4610.2005.05069.x

29. Neuhauser H, Leopold M, von Brevern M, Arnold G, Lempert T (2001) The interrelations of migraine, vertigo, and migrainous vertigo. Neurology 56:436-441. DOI: 10.1212/wnl.56.4.436

30. Oh AK, Lee H, Jen JC, Corona S, Jacobson KM, Baloh RW (2001) Familial benign recurrent vertigo. Am J Med Genet 100:287-291. DOI: 10.1002/ajmg.1294

31. Lee HK, Ahn SK, Jeon SY, Kim JP, Park JJ, Hur DG, Kim DW, Woo SH, Kang HS (2012) Clinical characteristics and natural course of recurrent vestibulopathy: a long-term follow-up study. Laryngoscope 122:883-886. DOI: 10.1002/lary.23188

32. Pan Q, Zhang Y, Zhang S, Wang W, Jiang H, Fan Y, Zhou J (2020) Clinical features and outcomes of benign recurrent vertigo: A longitudinal study. Acta Neurol Scand 141:374-379. DOI: 10.1111/ane.13214

\section{Figures}




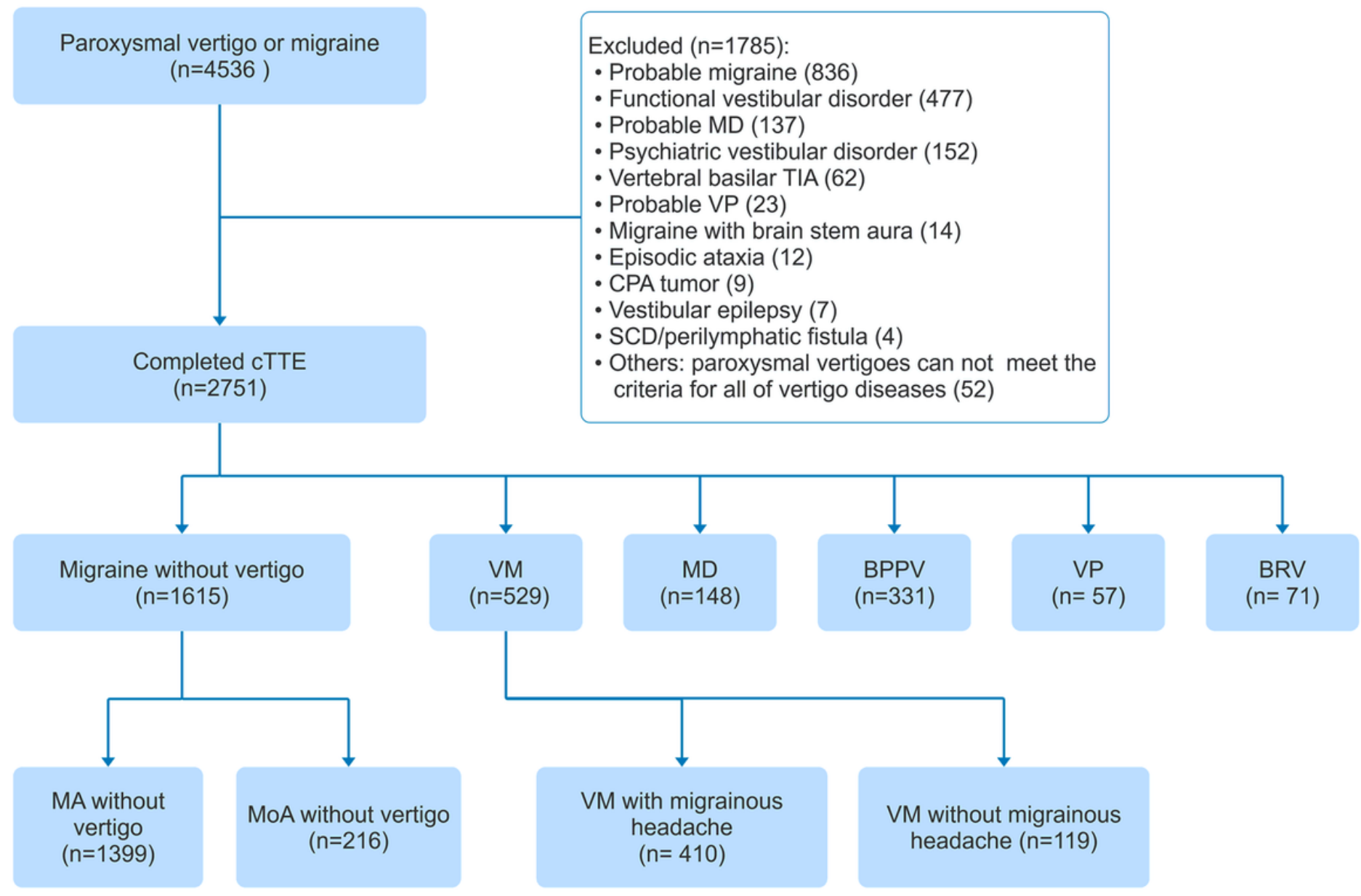

\section{Figure 1}

Flowchart of the inclusion and exclusion of patients 


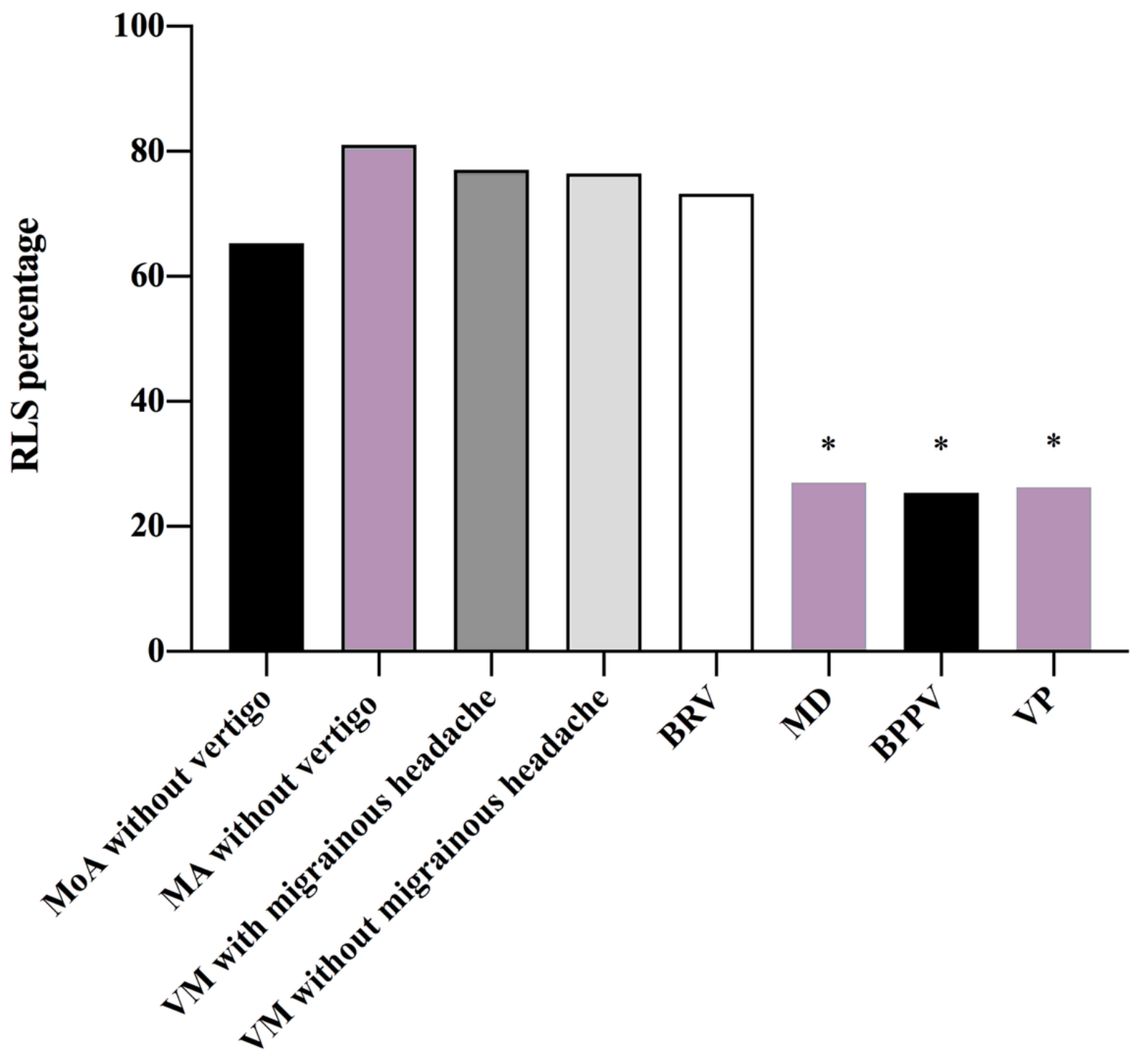

Figure 2

The frequency of RLS in patients with different diseases There is a statistical difference between the frequency of RLS in BRV patients and that in MD, BPPV and VP patients. The proportion of RLS in BRV patients is significantly higher than that in patients of MD, BPPV and VP $(P<0.05)$. The proportion of RLS in VM patients without migrainous headaches is also significantly higher than that in MD, BPPV and VP patients $(P<0.05)$. However, there is no statistical difference between the frequency of RLS in BRV patients and that in MoA patients $(P=0.931), M A(P=0.997)$, VM with migrainous headache $(P=0.787)$, and VM without migrainous headache ( $P=0.754)$. Abbreviations: $B P P V$, benign paroxysmal positional vertigo; BRV, benign recurrent vertigo; MA, migraine with aura; MD, Meniere's disease; MoA, migraine without aura; RLS, right-to-left shunt; VM, vestibular migraine; VP, vestibular paroxysmia 


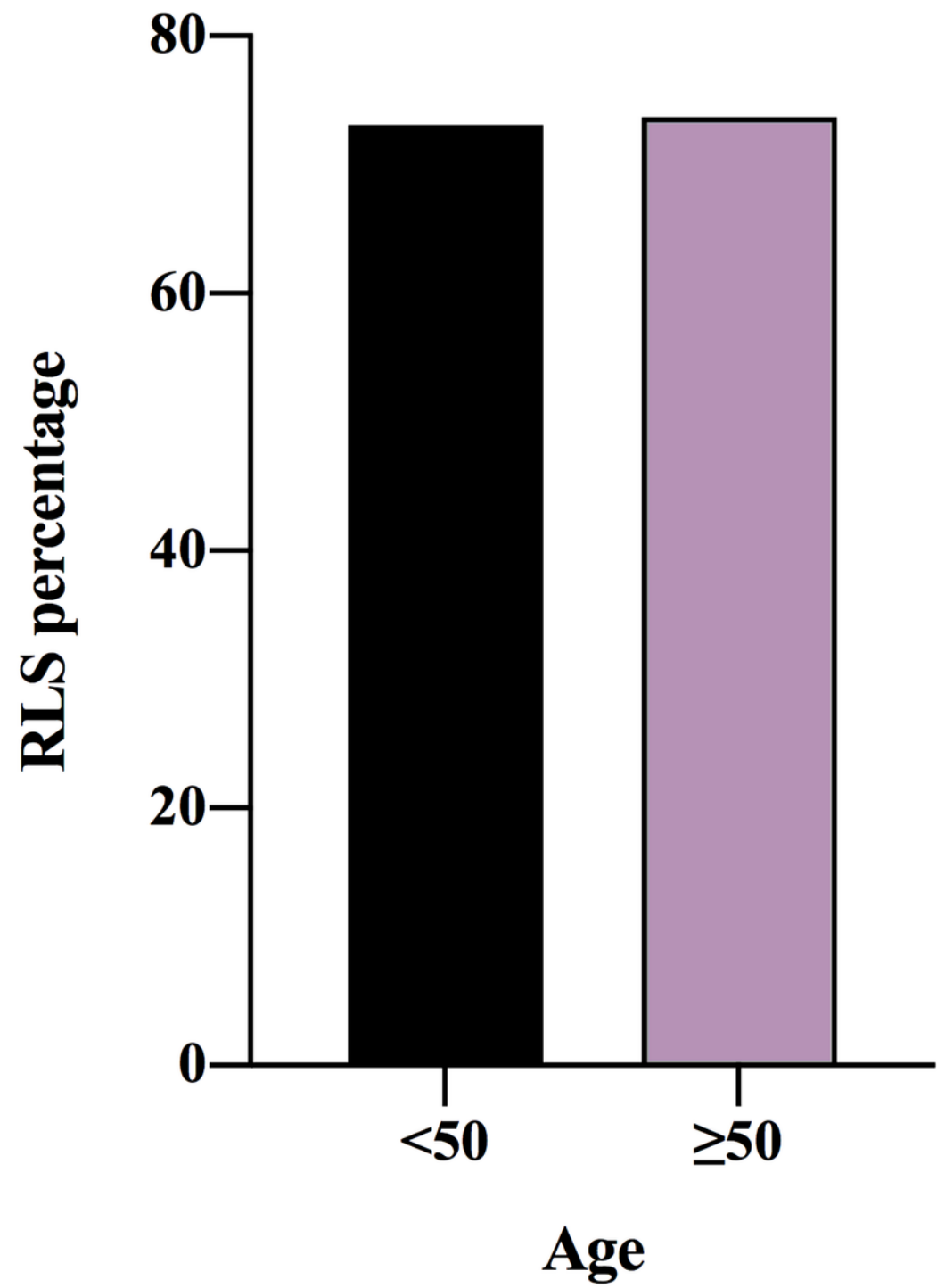

Figure 3

The frequency of RLS grouping by age in patients with BRV The proportion of RLS in BRV patients aged $<50$ years and those aged $\geq 50$ years was similar, and there was no statistical difference. Abbreviations: BRV, benign recurrent vertigo; RLS, right-to-left shunt 

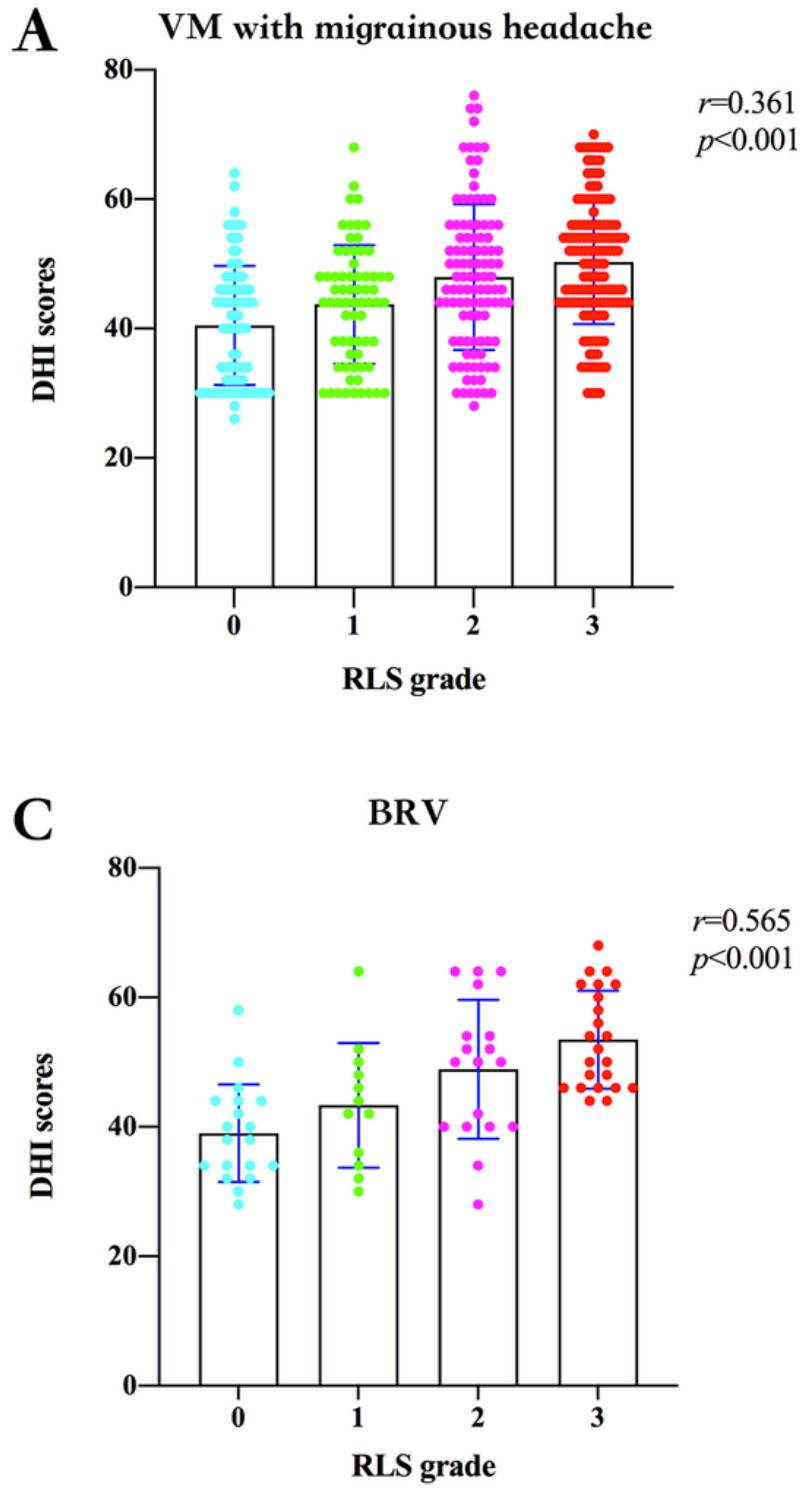
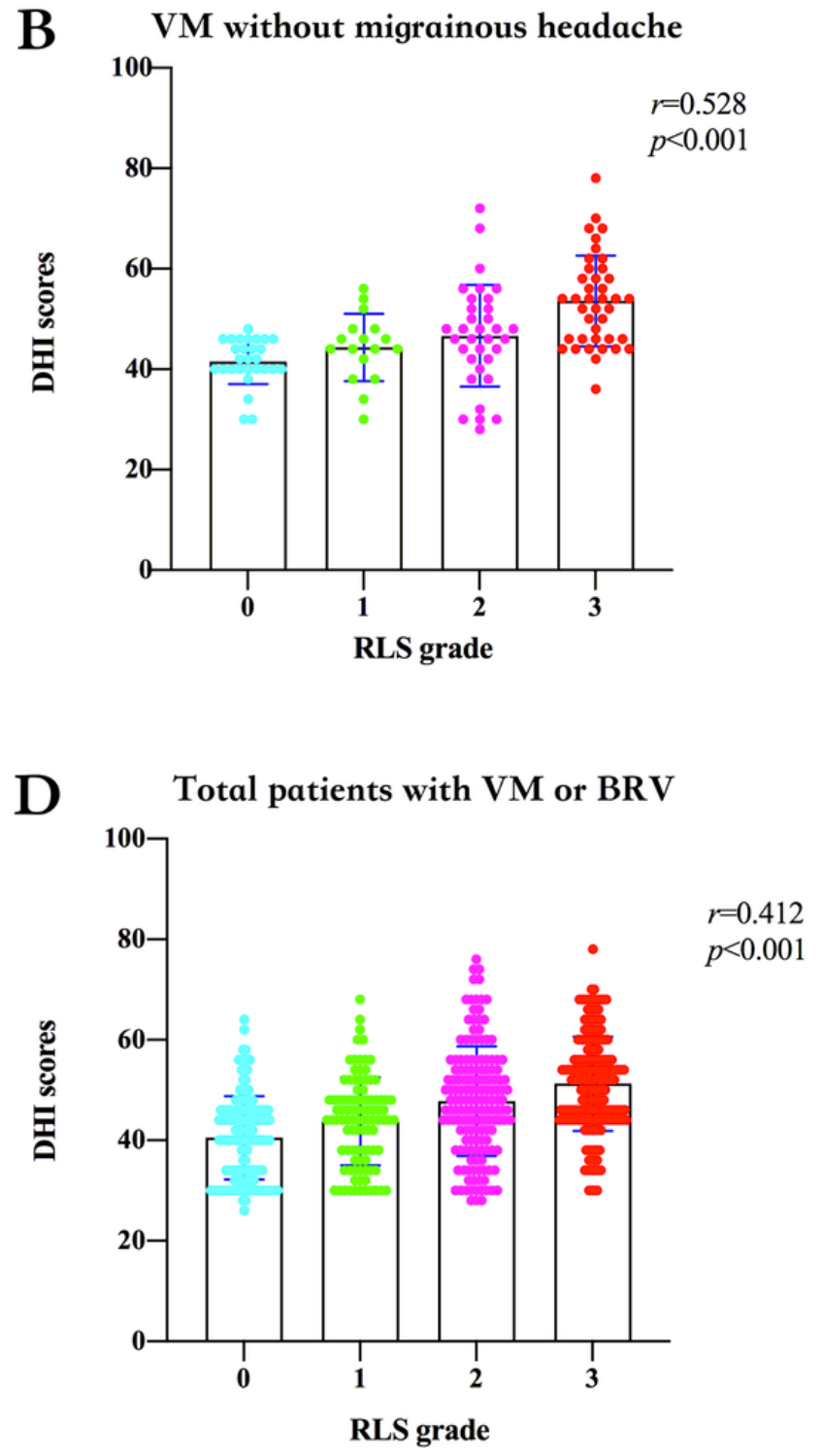

\section{Figure 4}

The DHI scores of RLS grade in patients with VM and BRV (A) In patients with VM with migrainous headache, DHI score increased with increasing RLS grade, and there was a positive correlation between them. (B) In patients with VM without migrainous headache, DHI score increased with increasing RLS grade, and there was a positive correlation between them. (C) In BRV patients, DHI score increased with increasing RLS grade, and there was a positive correlation between them. (D) In all patients with VM or BRV, the DHI score increased with increasing RLS grade, and there was a positive correlation between them. Abbreviations: DHI, Dizziness Handicap Inventory; RLS, right-to-left shunt; VM, vestibular migraine; BRV, benign recurrent vertigo 